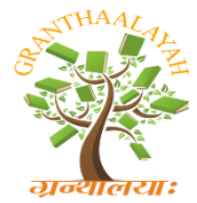

\author{
INTERNATIONAL JOURNAL OF RESEARCH - \\ GRANTHAALAYAH \\ A knowledge Repository
}

Science

\title{
EXPANDING THE ROLE OF MAGNETIC FIELDS IN RED BLOOD CELLS DEFORMATIONS: DEMONSTRATION OF PARAMAGNETIC AND DIAMAGNETIC FIELDS
}

\author{
Abraham A. Embi Bs *1 \\ ${ }^{* 1}$ Citizen Scientist, 13442 SW 102 Lane, Miami, Florida USA, 33186
}

\begin{abstract}
Background: The main purpose of this manuscript is to introduce a biophysics based mechanism of the role of magnetism in temporary cell deformation.

Biomagnetism: Biomagnetism has been slowly introduced in the medical literature as follows: The diamagnetic nature of blood reported in1936, the recording of organ emissions from the human body also published in the second half of the last century. It could stated that the same way molecular cross talk influence cells signaling, biomagnetic cross talk could also influence cells functions.

Introduction: This manuscript introduces the effect of externally applied magnetic fields (diamagnetic or paramagnetic) causing temporary Red Blood Cells (RBCs) deformations. Results from experiments using diamagnetic material such as human blood with the property of repelling an opposing magnetic field; and paramagnetic material such as carbon based graphite or iron particles are reported.

Methods: The work herein presented entailed the trapping (between two similar glass slides) of fresh human blood smear with fine exogenous metal, such as iron filings or graphite powder. The technique requires a standard video microscope and an image recording equipment. Provocative placement of ferromagnetic or diamagnetic material in in vitro blood smears preparations were found to exhibit temporary in vivo characteristics quoted Temporary In Vivo Blood Smears (TBIS). Established protocol calls for fresh blood smears to be set-aside 5 minutes prior to staining. During the first 2 minutes of the set-aside period, in a typical monolayer smear, one can observe an initial brief time period of approximately $1^{\prime} \pm 30$ " of a drying cycle. Usually, a moisture sheen boundary is seen gradually moving from the slide's edges towards the center.

Results: The main findings include a demonstration that both diamagnetic and pararmagnetic material deform RBCs in a variety of shapes. The paramagnetism (attraction) and diamagnetism of graphite powder induced temporary RBCs deformation in the form of teardrops. For the first time, an ongoing cellular deformation process was captured in video recordings.

Conclusions: When in vivo cells in blood smears, such as RBCs, are fronted by exogenous magnetic fields, temporary cell deformation occurs.
\end{abstract}

Keywords: Blood Smears; TBIS; Red Blood Cells Deformation; Optical Tweezers; Iron Paramagnetism; Graphite Paramagnetism. 
Cite This Article: Abraham A. Embi Bs. (2019). "EXPANDING THE ROLE OF MAGNETIC FIELDS IN RED BLOOD CELLS DEFORMATIONS: DEMONSTRATION OF PARAMAGNETIC AND DIAMAGNETIC FIELDS." International Journal of Research - Granthaalayah, 7(2), 214-220. https://doi.org/10.29121/granthaalayah.v7.i2.2019.1026.

\section{Introduction}

The main purpose of this manuscript is to introduce a biophysics based mechanism of the role of magnetic cross-talk causing temporary RBC deformation. The result of structural change causing cell deformation is herein reported as similar to RBCs deformations caused by the Optical Tweezers Trapping Approach (1)

\section{A Brief History of Biomagnetism}

Biomagnetism has been described as an "Interdisciplinary Approach" (2). To date, the field of biomagnetism has been gradually developed and introduced in the medical literature. For example, the diamagnetic nature of blood was introduced in 1936 (3), the recording of biomagnetic emissions from the human body ie: Brain, Heart and Hair follicles were also published in the 1960s; with a recent increase in publications $(4,5,6,7,8,9)$. In this manuscript, fresh whole blood drops were smeared onto a glass slide; that by conventional wisdom fits the in vitro definition; which is that of a process performed or taking place in a test tube, or elsewhere outside a living organism. This manuscript re-introduces a novel methodology where in vitro blood smears preparations are shown to exhibit transient in vivo characteristics, thus branded TIBS. Established protocol calls for fresh blood smears to be set-aside 5 minutes prior to staining. During the first 2 minutes of the set-aside period, in a typical monolayer smear, one can observe an initial brief time period of approximately $1 ' \pm 30$ " of a drying cycle. Usually, a moisture sheen boundary is seen gradually moving from the slide's edges towards the center. This is an indication of fluid evaporation. Provocative magnetic stimulations done during the sheen (transient) period of the fresh smears exhibited dynamic RBCs transformations and/or repulsive, attractive motion or a combined response; which in this author's opinion fits an in vivo biological change in an in vitro experiment $(10,11)$.

\section{Methods}

\section{Materials}

Clean 25x75x1mm glass slides, human blood drops, metal iron filings, powdered graphite, a video microscope Celestron Pentaview Model \#44348, and a McBook Pro Apple Computer with Photo Application and Power Point software capabilities for figures and video recording downloading, documentation, presentation and digital storage.

\section{Methods}

Blood smears were mounted according to established procedure (12)

The work herein presented entailed the placement of fine exogenous metal iron filings or fine graphite powder on a human blood smear while evaporation is taken place. Since time is the essence (Less that 20 seconds) to place the foreign particles on the evaporating smear, the following steps are suggested: 
- Preset the video microscope into the video mode.

- Have the exogenous particles easily accessible for placement.

- Puncture a finger digit and milk blood.

- Place two blood drops on a slide and prepare a fresh smear.

- Sprinkle the exogenous particles in the center of the evaporating smear.

- Cover the smear by an identical glass slide.

- Readily mount the smear on the microscope-viewing platform and locate suitable particle(s).

- Change the lens magnification for adequate details viewing.

- Begin video recording, with the goal of documenting the in vivo response.

- Select images for analysis and interpretation.

- Repeat above if unsuccessful.

\section{Results}

\section{Effect of Iron Static Ferromagnetic Fields on RBCs Deformation}

A total of $n=50+$ images of experiments are on file expressing RBCs induced iron filings RBCs deformations in a variety of teardrop shapes similar to the ones shown in a previous publication using low frequency magnetic fields (13). When metal iron filings were scattered on a blood smear slide, static and video recorded images show blood components being attracted towards the metal iron filings (Figure $1+$ video).

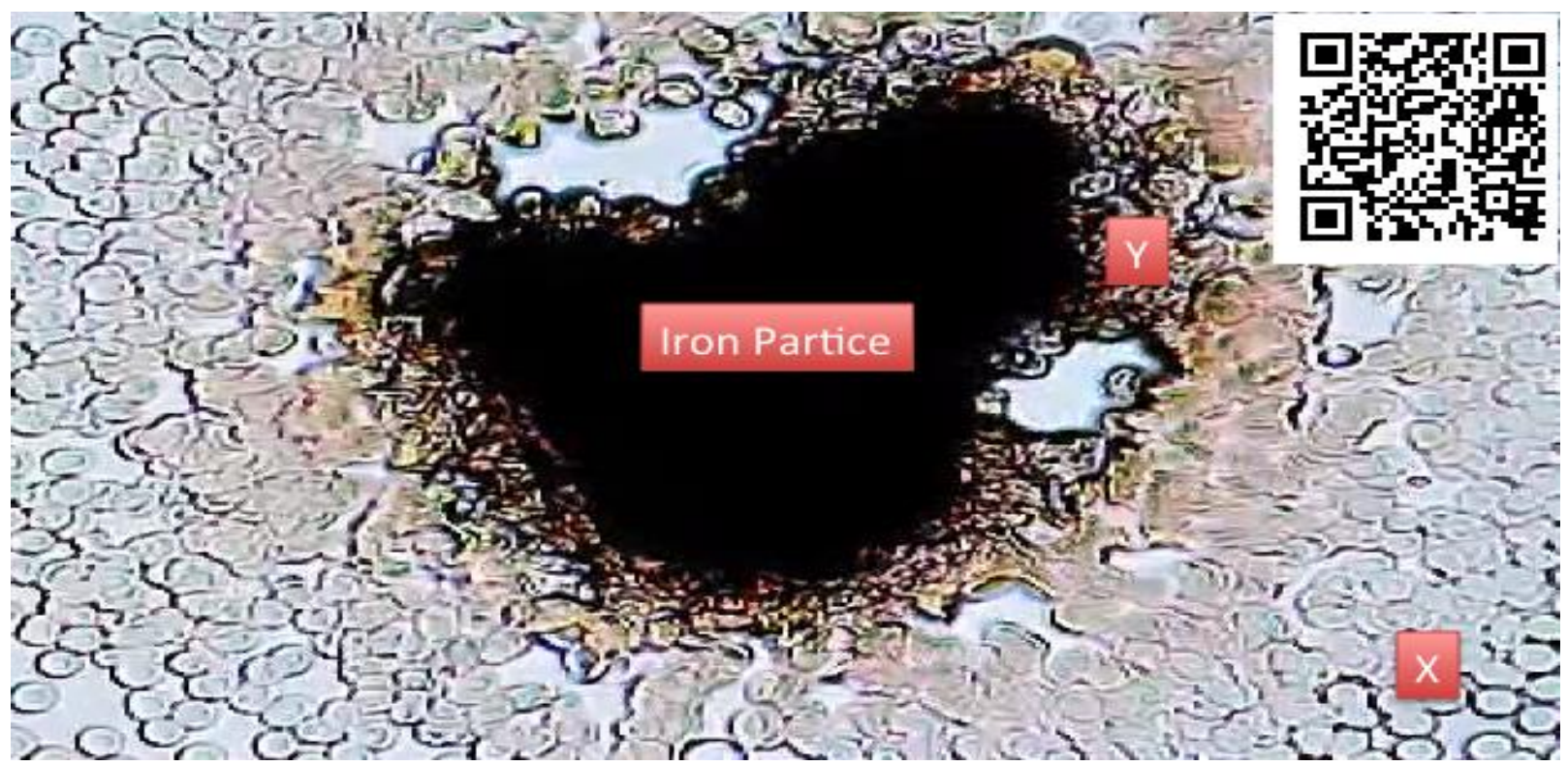

Figure 1: Microphotograph of video frame after moisture evaporation of a metal iron filing placed on blood smear during Temporary In vivo Blood Smear stage (TIBS). X= Blood smear. $\mathrm{Y}=$ Compressed RBCs by static magnetic attraction. For details visit https://youtu.be/5FOOZZ42nQ4 or scan QR Code on upper right image

Please observe in Figure 3 the "Not Deformed" (ND) labeled RBCs in video recording undergoing metamorphosis 


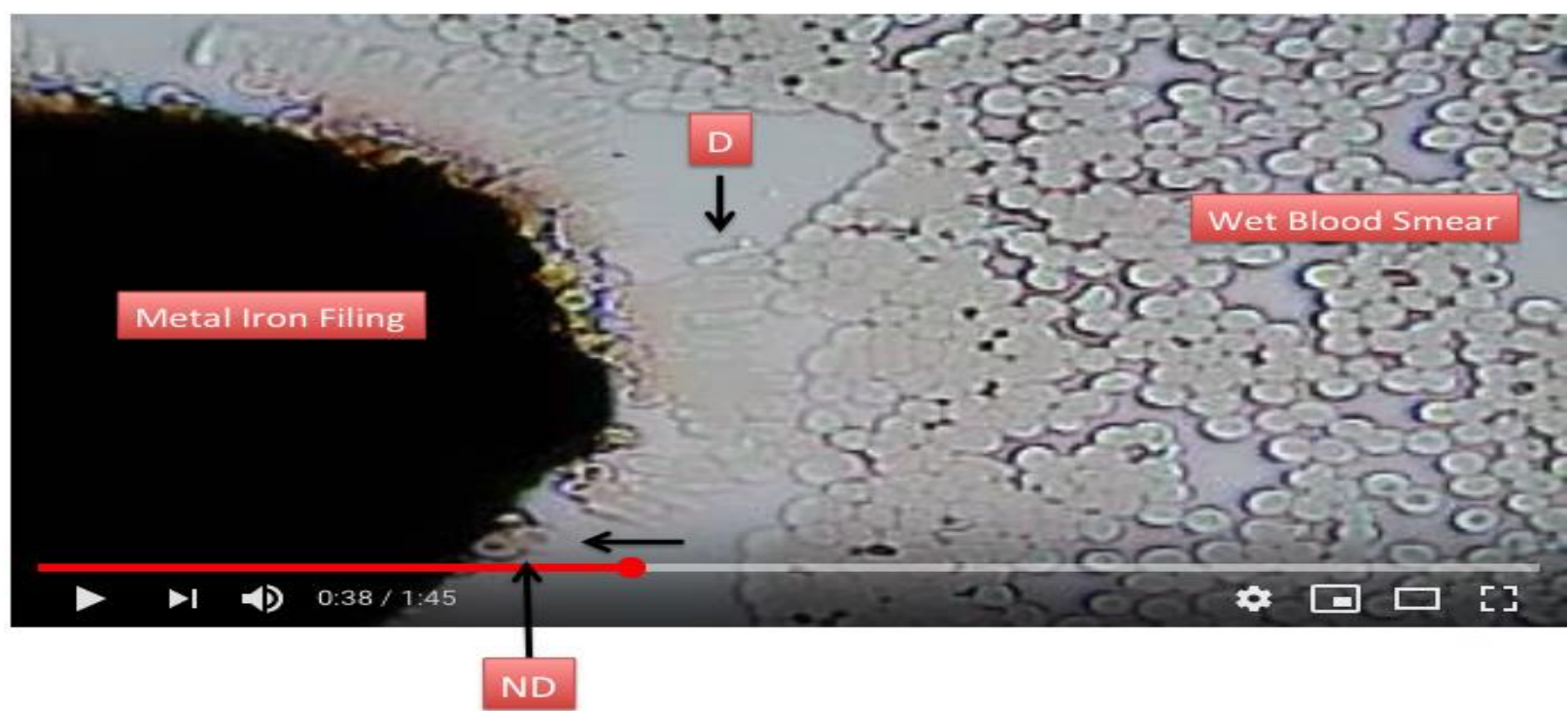

Figure 2: Video frame image at 0.38 " of video recording of Metal Iron Filing in in temporary vivo phase of blood in vitro smear (TBIS). $\mathrm{D}=$ Teardrop shape deformed $\mathrm{RBCs} . \mathrm{ND}=\mathrm{Control}$ $\mathrm{RBC}$

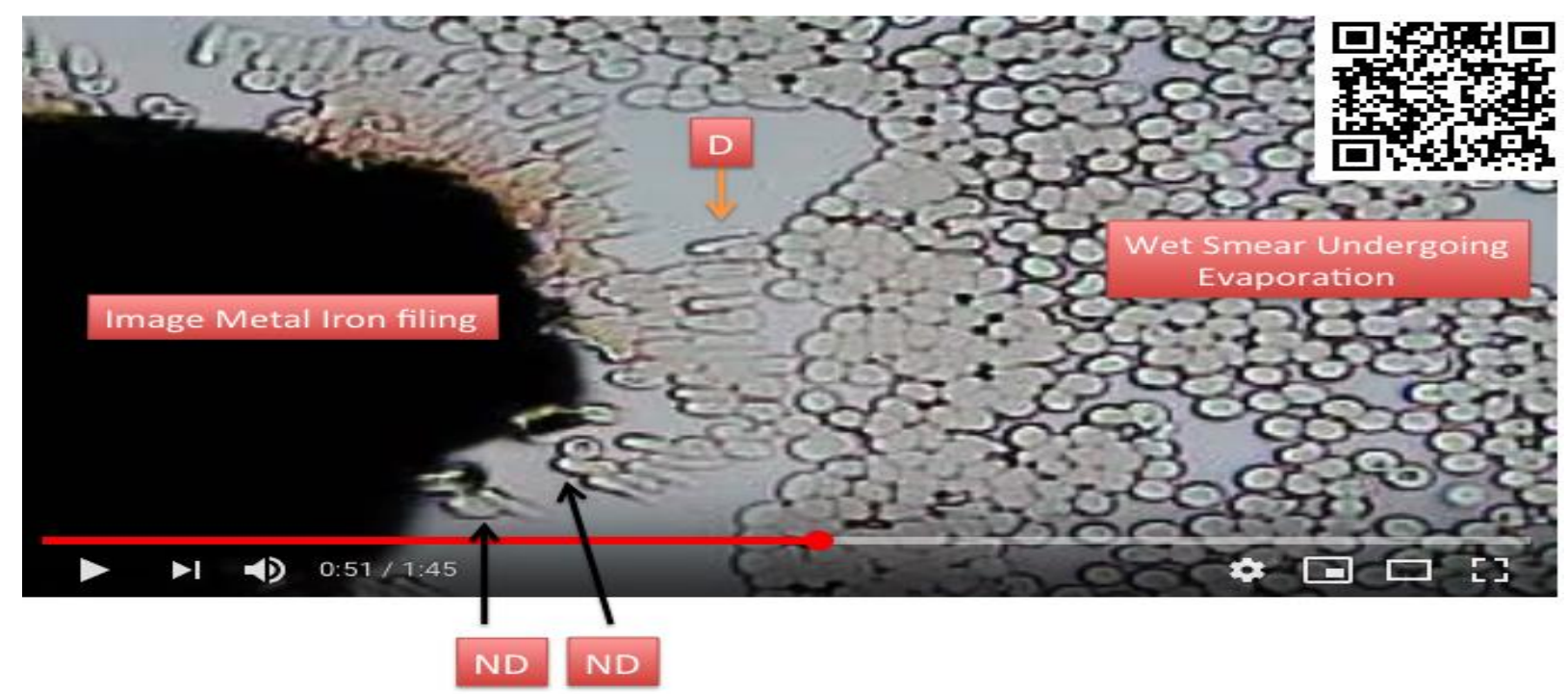

Figure 3: Video Frame at 0.51". Twenty three seconds later, Mechanism for genesis of RBCs shape deformations into teardrops on temporary vivo phase of blood in vitro smear (TBIS). $\mathrm{D}=$ Already Deformed RBCs. ND= RBCs to undergo metamorphosis. Please visit video recording at https://youtu.be/N1nDRm2-s5g or Scan QR code image (top right corner). To better appreciate the teardrop formation, suggest executing video replay repeatedly from frames 0.35 " forward and back guards to appreciate living quality of RBCs.

\section{The Graphite Experiments}

\section{Effect of Paramagnetic (attraction) of Powder Graphite on RBCs Deformation}

Published extensive investigations, documented the paramagnetic property of graphite, results were summarized as follows: "Our experiments show that the frequently reported ferromagnetism 
in pristine pyrolytic graphite is most likely to originate from contamination with Fe-rich inclusions introduced presumably during crystal growth" (14). In the present experiments, graphite powder was commercially obtained and tested as described in the methods section of this manuscript. Paramagnetism was also demonstrated in the graphite powder. The figure below complemented by video recording, shows the temporary RBCs structural changes,

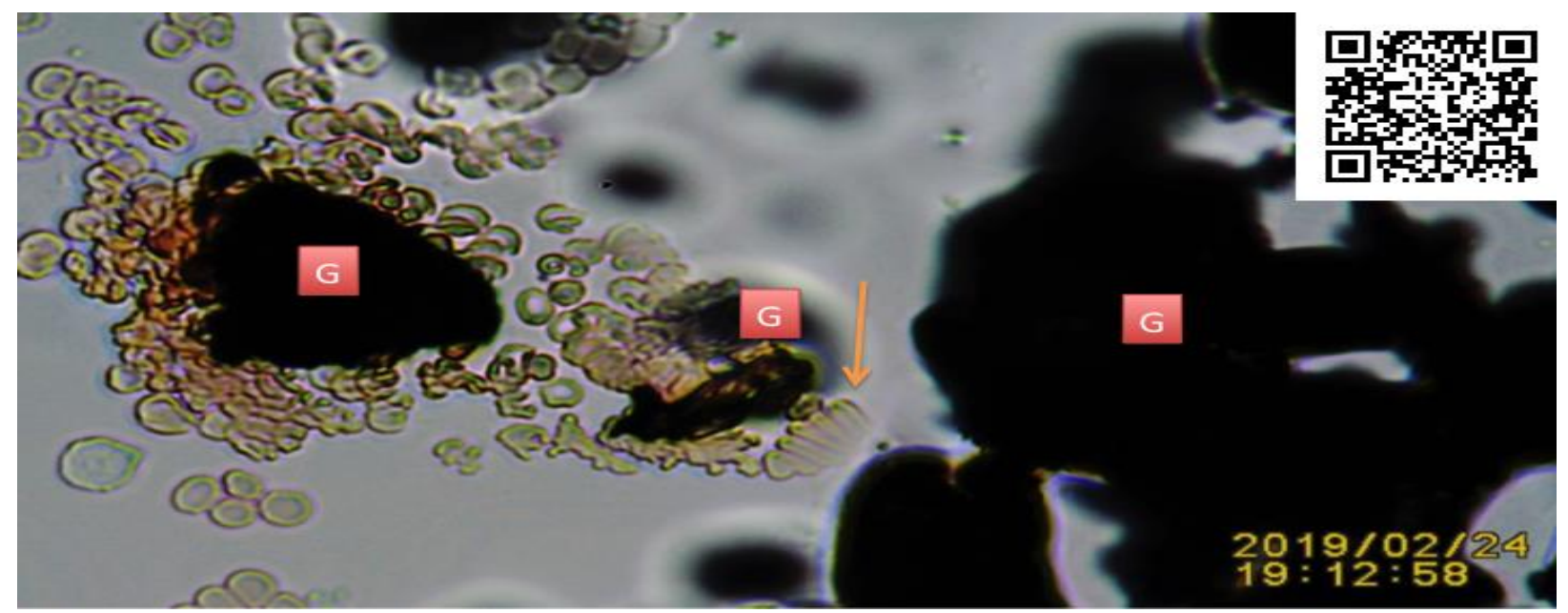

Figure 4: Microphotograph of RBCs in TIBS seen attracted and temporarily deformed as "teardrops" by the paramagnetic attraction of graphite powder.

$\mathrm{G}=$ Graphite Powder. Orange Arrow= Deformed RBCs.

For further details, please refer to link: https://youtu.be/jvGJtinz2Sw or Scan QR Code on top right corner of image

\section{Effect of Diamagnetic Graphite in TIBS Blood Smear}

Graphite fragments from a pencil tip, showed diamagnetic properties (repulsion of external field). This phenomenon is documented below, concurrent with inducing RBCs deformations.

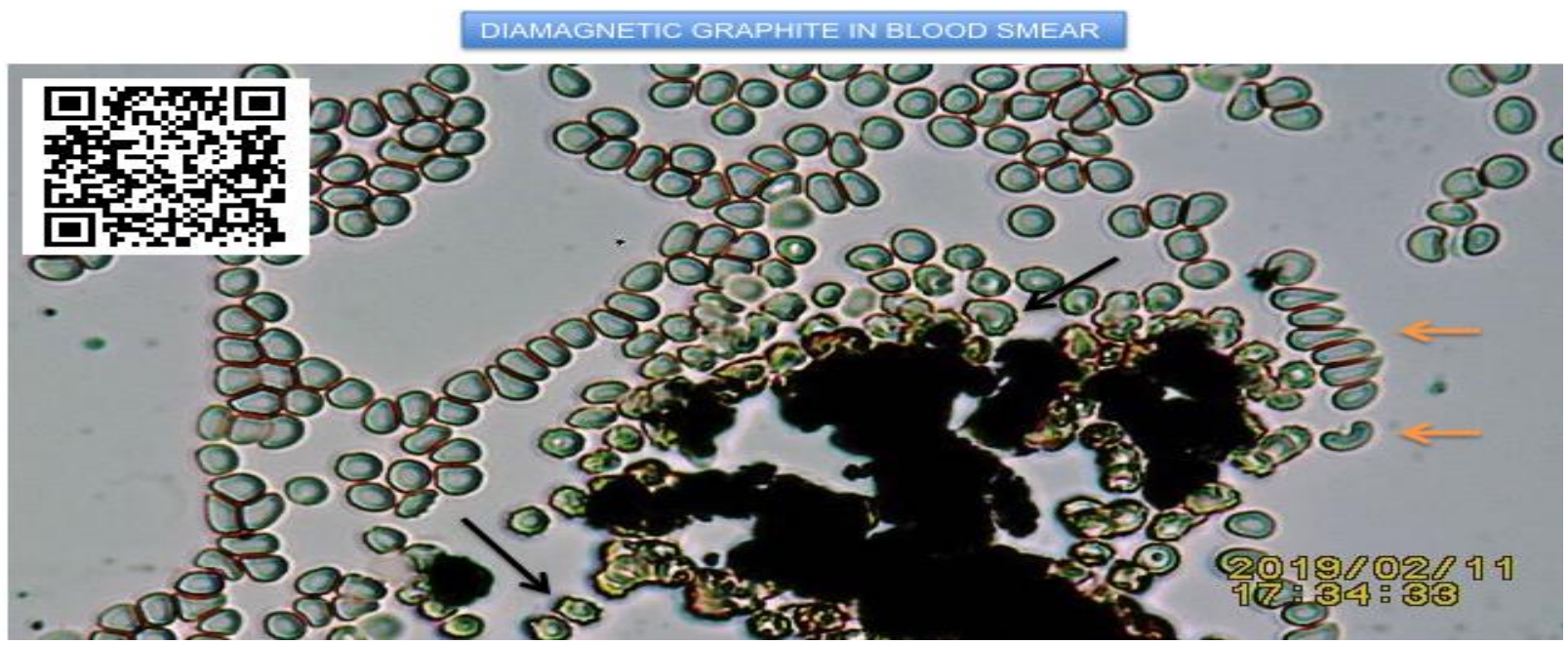

Figure 5: Diamagnetic graphite fronting diamagnetic blood. Microphotograph of last frame at 00.50" (end of video) showing: Orange Arrow: Deformed RBC. Black Arrow= Crenocytes clumping adjacent to graphite. To view video recording click on link below https://youtu.be/VTQf2MPIEtI or Scan QR Code on left upper side of figure. 


\section{Discussion}

\section{Magnetic Fields as Primary Factor in Temporary RBCs Deformation}

The discovery of temporary in vivo characteristics in an in vitro blood smear has allowed for the introduction of a tabletop microscopy method documenting magnetic fields (read forces) as primary mechanisms inducing red blood cells deformations. Two types of magnetic materials were evaluated, the ferromagnetic metal iron filings and ferromagnetic graphite powder.(Fig. $3+$ video) and (Fig. $4+$ video). Both causing RBCs deformations. This author suggests that the mechanism causing the cell deformations is due to the fronting of magnetic fields. In the case of iron paramagnetic forces are fronted with the blood diamagnetism. The attraction of paramagnetism applied to RBCs trapped between graphite particles is clearly demonstrated in Figure $4+$ video recording. RBCs are seen attracted and adhering to graphite, then subsequently changing shape due to a greater magnetic pulling (by a larger particle) causing dislodging and continuing their migration.

\section{Summary and Conclusions}

The findings herein reported goes beyond the concept of In vitro to in vivo extrapolation; which refers to the qualitative or quantitative transposition of experimental results or observations made in vitro to predicts phenomena in vivo in biological organisms (Wikipedia). In this manuscript there is no extrapolation, cells are demonstrated undergoing actual temporary changes in video recordings from exposure to two paramagnetic materials, such as metal iron filings and fine graphite powders.

This manuscript introduces a novel methodology that allows for the study of living non-dehydrated blood tissue on a glass smear. This in vivo phase in an in vitro glass smear preparation lasting on average 1' \pm 30 " was dubbed a Temporary In Vivo Blood Smear or (TIBS). A conclusion of magnetism as a primary factor in cell deformation is supported by the data herein presented.

\section{Limitations}

It could be argued that the blood tissue studied was from one subject (the author); therefore having little or no statistical relevancy.

Additional Relevant Information:

Two instances mitigate the limitation:

1) In a prior published paper, blood drops tested on three other volunteers (one male and two females) showed similar RBCs deformations (REF).

2) Since all blood drops studies were from one person (the author), a standard cell blood count including a pathologist review of the author's blood smear was done during a routine medical visit. The test was done February $12^{\text {th }} 2019$ at a reputable community hospital. Today is February $15^{\text {th }} 2019$. There was no mention of any deformed RBCs in the form of Dacrocytes (Teardrop). Those official findings finding support the data and conclusions presented in the manuscript. Findings available upon request. 


\section{References}

[1] Li J, Dao M, Lim CT, Suresh S. 2005 Spectrin-level modeling of the cytoskeleton and optical tweezers stretching of the erythrocyte. 2005 Biophys J. 2005 May;88(5):3707-19. PMID: 5749778 DOI: 10.1529/biophysj.104.047332

[2] Williamson, S.J., Romani, G.-L., Kaufman, L., Modena, I. 1983 Biomagnetism An Interdisciplinary Approach. eBook. ISBN 978-1-4757-1785-3 Springer. DOI 10.1007/978-1-47571785-3 (Online) 9781475717877 (print)

[3] Pauling, L., \& Coryell, C. D. 1936. The Magnetic Properties and Structure of Hemoglobin, Oxyhemoglobin and Carbonmonoxyhemoglobin. Proceedings of the National Academy of Sciences of the United States of America, 22(4), 210-6

[4] Baule G.M, McFee R. 1963 Detection of the magnetic field of the heart. American Heart Journal ;66: 95-96 PBMID: 14045992.

[5] Cohen D. 1972 Magnetoencephalography: Detection of the Brain's electrical activity with a superconducting magnetometer. Science ;175: 664-666 PMID: 5009769.

[6] Corsini E, Acosta V, Baddour N, Higbe J, Lester B, Licht P, Patton B, Prouty M, Budker D. 2011 Search for plant biomagnetism with a sensitive atomic magnetometer. J Appl Physics.;109: 07470$1-5$.

[7] Hong, F.T. 1995. Magnetic field effects on biomolecules, cells, and living organisms. Bio Systems, $363,187-229$.

[8] Scherlag BJ, Huang B, Zhang L, Sahoo K, Towner R, Smith N, Embi AA, Po SS. 2015 Imaging the Electromagnetic Field of Plants (Vigna radiata) Using Iron Particles: Qualitative and quantitative correlates. Journal of nature and Science ;1: e61.

[9] Embi AA, Jacobson JI, Sahoo K, Scherlag BJ Demonstration of Inherent Electromagnetic Energy Emanating from Isolated Human Hairs. Journal of Nature and Science 2015; 1: e55.

[10] Quignot N., Hamon J., Bois F., 2014, Extrapolating in vitro results to predict human toxicity, in In Vitro Toxicology Systems, Bal-Price A., Jennings P., Eds, Methods in Pharmacology and Toxicology series, Springer Science, New York, USA, p. 531-550

[11] Wilk-Zasadna, I., Bernasconi, C., Pelkonen, O., \& Coecke, S. (2015). Biotransformation in vitro: An essential consideration in the quantitative in vitro-to-in vivo extrapolation (QIVIVE) of toxicity data. Toxicology, 332, 8-19 https://doi.org/10.1016/j.tox.2014.10.006

[12] Center for Disease Control. USA. Suggested technique for BloodSmear. https://www.cdc.gov/dpdx/resources/pdf/benchAids/malaria/Malaria_procedures_benchaid.pdf.

[13] Embi A. (2018). "BIOMAGNETISM AS FACTOR IN RED BLOOD CELLS DEFORMATION." International Journal of Research - Granthaalayah, 6, 46-57. https://doi.org/10.5281/zenodo.2528404

[14] M. Sepioni, R. R. Nair, I.-Ling Tsai, A. K. Geim and I. V. Grigorieva (2012) Revealing common artifacts due to ferromagnetic inclusions in highly oriented pyrolytic graphite. Europhysics Letters, Volume 97, Number 4. DOI:

*Corresponding author.

E-mail address: embi21@ att.net 